\title{
Perinuclear Cisternal Space
}

National Cancer Institute

\section{Source}

National Cancer Institute. Perinuclear Cisternal Space. NCI Thesaurus. Code C33303.

The area between the inner and outer nuclear membranes that is contiguous with the lumen of the rough endoplasmic reticulum. 